\title{
Long non-coding RNA LINC00152 promotes cell proliferation, metastasis, and confers 5-FU resistance in colorectal cancer by inhibiting miR-139-5p
}

Zehua Bian', Jiwei Zhang ${ }^{1}$, Min Li ${ }^{1}$, Yuyang Feng ${ }^{1}$, Surui Yao', Mingxun Song ${ }^{1}$, Xiaowei Qi ${ }^{2}$, Bojian Fei ${ }^{3}$, Yuan Yin ${ }^{1}$, Dong Hua ${ }^{1,4}$ and Zhaohui Huang ${ }^{1}$

\begin{abstract}
Long intergenic non-coding RNA 152 (LINCO0152) is a recently identified tumor-promoting long non-coding RNA. However, the biological functions of LINC00152 in colorectal cancer (CRC) remain unclear and require further research. The aim of the present study is to explore the roles of LINC00152 in cellular function and its possible molecular mechanism. In this study, we discovered that LINC00152 was overexpressed in CRC tissues and negatively related to the survival time of CRC patients. Functional analyses revealed that LINCO0152 could promote cell proliferation. Furthermore, LINC00152 could increase the resistance of CRC cells to 5-fluorouracil (5-FU) by suppressing apoptosis. We also discovered that LINCO0152 could enhance cell migration and invasion. Mechanistic studies demonstrated that LINC00152 could regulate the expression of NOTCH1 through sponging miR-139-5p and inhibiting its activity from promoting CRC progression and development. Altogether, our work points out a novel LINC00152/miR-139-5p/ $\mathrm{NOTCH} 1$ regulatory axis in CRC progression and development.
\end{abstract}

\section{Introduction}

Colorectal cancer (CRC) is the third most common cancer worldwide ${ }^{1}$. The occurrence and development of CRC involve a series of complex changes at the genetic and epigenetic levels ${ }^{2}$. Increasing number of studies have demonstrated that long non-coding RNAs (lncRNAs) are involved in the occurrence and development of $\mathrm{CRC}^{3}$.

LncRNAs are a kind of RNA molecules with more than 200 nucleotides and no protein translation ability. Recent advances have revealed the vital roles of IncRNAs in regulating tumorigenesis, and progression. Long intergenic non-coding RNA 152 (LINC00152) locates on

\footnotetext{
Correspondence: Zhaohui Huang (hzhwxsy@126.com)

${ }^{1}$ Wuxi Cancer Institute, Affiliated Hospital of Jiangnan University, Wuxi, Jiangsu 214062, China

${ }^{2}$ Department of Pathology, Affiliated Hospital of Jiangnan University, Wuxi,

Jiangsu 214062, China

Full list of author information is available at the end of the article
}

chromosome 2p11.2 with $828 \mathrm{nt}$ transcription length. LINC00152 was overexpressed in tumor tissues and plasma of gastric cancer (GC) patients, and could promote GC cell proliferation and cell cycle progression through regulating EGFR and EZH2 $2^{4-7}$. LINC00152 also plays an oncogenic role in liver ${ }^{8}$, gallbladder ${ }^{9}$, and lung cancer $^{10}$. In addition, LINCO0152 is likely to be an indicator of stress in a variety of cells ${ }^{11}$. These studies exhibit the key oncogenic role and complicated mechanisms of LINC00152 in cancers. However, the detailed functions and mechanisms of LINC00152 in CRC are mainly unclear.

In this study, we showed that LINC00152 was upregulated in CRC, and correlated with poor survival. Functional analyses showed that LINCO0152 could enhance CRC growth, metastasis, and chemoresistance. Mechanistic studies demonstrated that LINC00152 promotes tumorigenesis and progression via working as a 

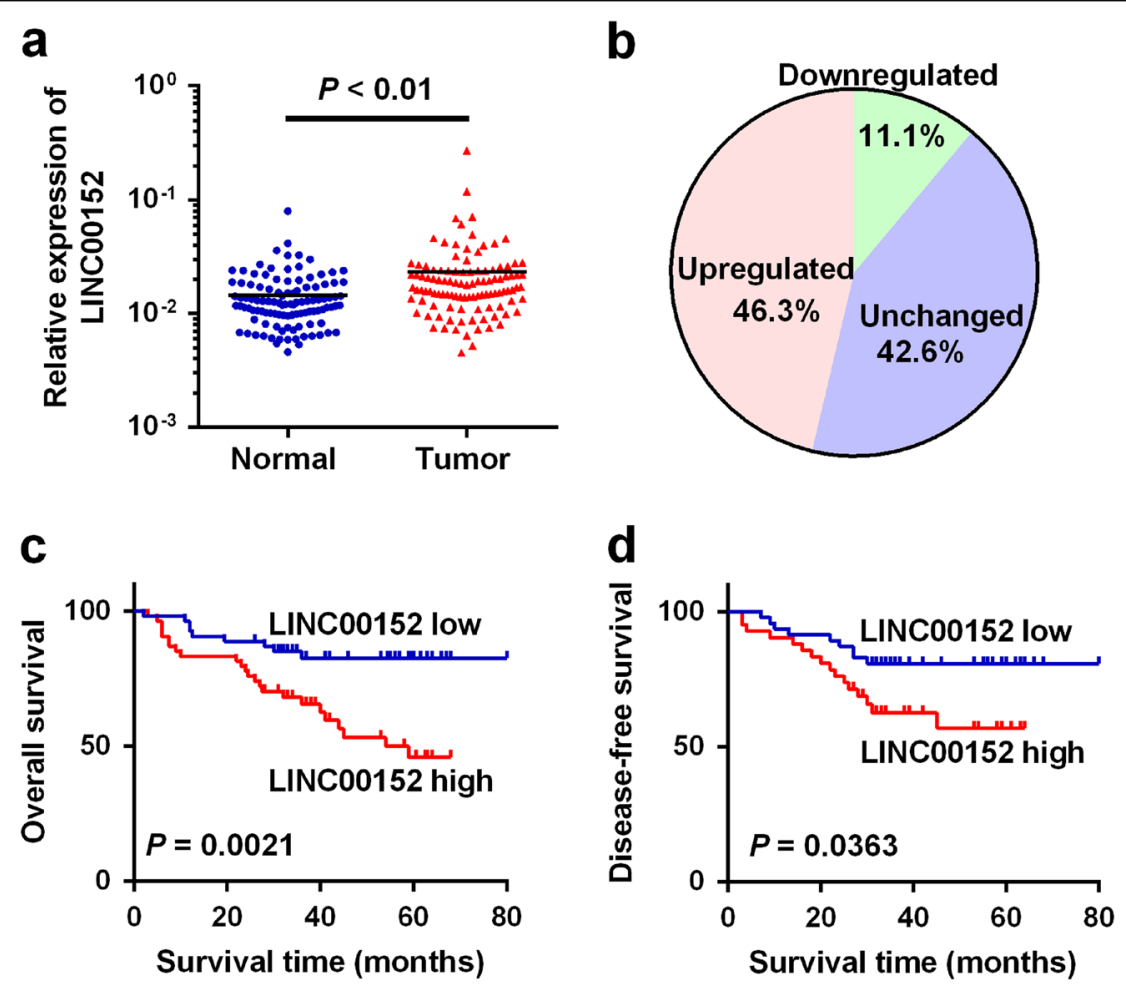

Fig. 1 LINC00152 is upregulated in tumor tissues of CRC. a Relative expression levels of LINC00152 in 108 paired CRC and NCTs were quantified by qRT-PCR. b LINC00152 was upregulated (> 2-fold) in $46.3 \%$ of the CRC tissues compared with the NCTs. c, d Kaplan-Meier survival analysis of the overall survival and disease-free survival in two groups defined by low and high expression of LINC00152 in patients with CRC

competitive endogenous RNA (ceRNA) of miR-139-5p, which is a key tumor suppressive microRNA (miRNA) ${ }^{12-}$ 18 . The present work reveals a novel regulatory pathway of LINC00152/miR-139-5p/NOTCH1 in CRC, suggesting that LINC00152 is a new prognostic factor and potential therapeutic target in CRC.

\section{Results}

Overexpression of LINC00152 in CRC associates with poor prognosis

To study the role of LINC00152 in CRC, we first detected its expression in 108 paired CRC tissues and noncancerous tissues (NCTs). The results revealed that LINC00152 was obviously upregulated in CRC $(P<0.001$, Fig. 1a), and $46.3 \%$ (50 of 108) of the CRC tissues showed $>2$-fold upregulation of LINC00152 compared with their NCTs (Fig. 1b).

To assess the potential association of LINC00152 with clinicopathological features, we first divided the 108 patients into LINC00152-high and -low groups. We found that the LINCO0152 levels in CRCs were significantly correlated with tumor stage $(P=0.013)$, whereas no obvious correlation between LINC00152 expression and other clinicopathological parameters was observed (Table 1).
The survival analysis showed that patients in the LINC00152-high group showed a shorter survival time than those in the LINC00152-low group (46.614 \pm 3.366 vs. $69.338 \pm 3.271$ months; log rank $=9.456, P=0.0021$, Fig. 1c). In addition, high LINC00152 expression was also associated with poor disease-free survival (log rank= 4.383, $P=0.0363$, Fig. 1d). Furthermore, multivariate analysis further identified that LINC00152 was an independent prognosis factor for CRC (hazard ratio (HR) = $2.514,95 \%$ confidence interval $(\mathrm{CI})=1.125-5.621, P=$ 0.025 , Table 2).

\section{LINC00152 promotes CRC cell proliferation}

The expression analyses of LINC00152 in six CRC cell lines showed that LoVo and SW480 have relatively high expressions of LINC00152, whereas HCT116 and HT29 have relatively low expressions of LINC00152 (Fig. 2a). To investigate the biological functions of LINC00152 in CRC, we overexpressed LINC00152 in HCT116 and HT29 cells, and inhibited LINC00152 expression in LoVo and SW480 cells (Fig. 2b). We observed that LINC00152 overexpression significantly promoted CRC cell proliferation and colony formation. In contrast, decreased cell growth, and colony formation abilities were showed in 
Table 1 Correlation of the expression of LINC00152 with clinicopathologic features

\begin{tabular}{|c|c|c|c|}
\hline \multirow[t]{2}{*}{ Characteristics } & \multicolumn{2}{|c|}{ LINC00152 } & \multirow[t]{2}{*}{$P$-value } \\
\hline & Low & High & \\
\hline \multicolumn{4}{|l|}{ Ages (years) } \\
\hline$<60$ & 30 & 30 & 1.000 \\
\hline$\geq 60$ & 24 & 24 & \\
\hline \multicolumn{4}{|l|}{ Gender } \\
\hline Male & 31 & 33 & 0.434 \\
\hline Female & 23 & 21 & \\
\hline \multicolumn{4}{|l|}{ Tumor size (cm) } \\
\hline$<5$ & 42 & 40 & 0.552 \\
\hline$\geq 5$ & 12 & 14 & \\
\hline \multicolumn{4}{|l|}{ Location } \\
\hline Colon & 30 & 29 & 0.847 \\
\hline Rectum & 24 & 25 & \\
\hline \multicolumn{4}{|l|}{ Differentiation } \\
\hline Well and moderately & 48 & 44 & 0.283 \\
\hline Poorly & 6 & 10 & \\
\hline \multicolumn{4}{|l|}{ Depth of tumor } \\
\hline $\mathrm{T} 1+\mathrm{T} 2$ & 13 & 6 & 0.083 \\
\hline $\mathrm{T} 3+\mathrm{T} 4$ & 41 & 48 & \\
\hline \multicolumn{4}{|l|}{ Distant metastasis } \\
\hline Absent & 47 & 48 & 0.768 \\
\hline Present & 7 & 6 & \\
\hline \multicolumn{4}{|l|}{ Tumor stage } \\
\hline $1+\|$ & 31 & 16 & 0.013 \\
\hline$I I I+I V$ & 23 & 38 & \\
\hline
\end{tabular}

LINC00152-silenced cells (Fig. 2c-e). Furthermore, ectopic LINC00152 expression promoted CRC tumor growth in vivo (Fig. 2f). All these data reveal the growthstimulating functions of LINC00152 in CRC.

\section{LINC00152 promotes cell cycle progression and confers resistance to 5 -FU-induced apoptosis}

To investigate the mechanism mediating the growthpromoting functions of LINC00152 in CRC, we measured the cell cycle distribution in the LINC00152-overexpressed and silenced CRC cells. As shown in Fig. 3a, ectopic LINC00152 expression resulted in an increased number of cells in S phase, whereas LINCO0152 knockdown caused a decreased cell number in S phase, indicating the promotion of the cell cycle by LINC00152.

5-fluorouracil (5-FU) is a basic drug for CRC treatment, and we evaluated the effect of LINCO0152 on 5-FU sensitivity in CRC cells. After overexpression or knockdown of LINC00152, CRC cells were then assayed for their sensitivity to 5 -FU by a CCK- 8 assay. The results showed that ectopic LINC00152 expression decreased the sensitivity of HCT116 cells to 5-FU, whereas LINC00152 silencing increased the sensitivity to 5-FU in LoVo cells (Fig. 3b). Given the key role of apoptosis in cancer chemotherapy, we further measured the effect of LINC00152 on 5-FU-induced apoptosis. The results showed that the LINC00152 overexpression significantly antagonize 5-FU-induced apoptosis, whereas the LINC00152 knockdown could augment apoptosis caused by 5 -FU (Fig. 3c).

\section{LINC00152 promotes CRC cell migration and invasion}

Transwell assays were then performed to measure the impact of LINC00152 on CRC metastasis. We observed that ectopic LINC00152 expression significantly facilitated migration and invasion in HCT116 cells (Fig. 4a), whereas the LINC00152 knockdown suppressed migration and invasion in LoVo cells (Fig. 4b).

\section{LINC00152 sponges miR-139-5p}

To investigate underlying mechanisms of LINC00152 in CRC, we first measured the subcellular localization of LINC00152 in HCT116 cells, and revealed that LINC00152 was localized predominantly in the cell cytoplasm (Fig. 5a), suggesting that LINC00152 may regulate tumorigenesis at the post-transcriptional level. LncRNAs could act as molecular sponges to modulate mRNAs expression by competitively binding their common miRNA responsive elements (MREs). Previous studies have proved that LINCO0152 could function as a ceRNA in human cancers ${ }^{19-21}$. We hypothesized that LINC00152 could promote CRC tumorigenesis and progression by suppressing the functions of certain miRNAs. Based on the bioinformatics analysis and Xia's work ${ }^{22}$, we found that LINC00152 harbors a recognition sequence of miR-139-5p (Fig. 5b). In view of the opposite functions of miR-139-5p and LINC00152 in CRC ${ }^{12-18}$, we intended to explore the potential relationship between them in CRC.

We first constructed reporter vectors containing LINC00152 (pLuc-LINC00152-WT) or its mutant with mutations in the seed sequence of miR-139-5p (pLucLINC00152-Mut), and then evaluated this underlying correlation of miR-139-5p with LINC00152 using luciferase reporter assays. We observed that miR-139-5p overexpression led to a marked inhibition in the reporter activity of pLuc-LINC00152-WT compared with that of pLuc-LINC00152-Mut (Fig. 5c), suggesting sequencespecific binding and inhibition of LINCO0152 by miR139-5p. To further validate the potential binding of LINC00152 to miR-139-5p, an RNA Immunoprecipitation (RIP) assay using an anti-Ago2 antibody was performed. The data exhibited that both LINC00152 and miR-139-5p 
Table 2 Univariate and multivariate regression analyses of parameters associated with prognosis of CRC patients

\begin{tabular}{|c|c|c|c|c|c|}
\hline \multirow[t]{2}{*}{ Characteristics } & \multirow[t]{2}{*}{ Subset } & \multicolumn{2}{|c|}{ Univariate analysis } & \multicolumn{2}{|c|}{ Multivariate analysis } \\
\hline & & $P$-value & HR $(95 \% \mathrm{Cl})$ & $P$-value & HR $(95 \% \mathrm{Cl})$ \\
\hline Ages (years) & $<60 / \geq 60$ & 0.421 & $0.747(0.367-1.519)$ & - & - \\
\hline Gender & Male/female & 0.818 & $0.921(0.458-1.853)$ & - & - \\
\hline Tumor size & $<5 \mathrm{~cm} / \geq 5 \mathrm{~cm}$ & 0.385 & $1.355(0.683-2.691)$ & - & - \\
\hline Location & Colon/rectum & 0.965 & $1.016(0.512-2.016)$ & - & - \\
\hline Differentiation & Well + moderately/poorly & 0.019 & $2.499(1.160-5.384)$ & 0.061 & $2.103(0.967-4.573)$ \\
\hline Depth of tumor & $\mathrm{T} 1+\mathrm{T} 2 / \mathrm{T} 3+\mathrm{T} 4$ & 0.035 & $8.471(1.157-62.029)$ & 0.169 & $4.083(0.549-30.370)$ \\
\hline Distant metastasis & Present/absent & 0.404 & $0.602(0.182-1.986)$ & - & - \\
\hline Tumor stage & $I+\|/\| I I+I V$ & 0.000 & $10.017(3.054-32.862)$ & 0.001 & $7.140(2.155-23.656)$ \\
\hline LINC00152 & High/low & 0.001 & $3.825(1.723-8.493)$ & 0.025 & $2.514(1.125-5.621)$ \\
\hline
\end{tabular}

were obviously enriched in Ago2 complex, demonstrating that LINC00152 is included in miRNPs, probably through binding with miR-139-5p (Fig. 5d).

\section{LINC00152 modulates NOTCH1 expression by competitively binding miR-139-5p}

Previous studies have shown that miR-139-5p inhibit CRC tumorigenesis, development, and chemoresistance by regulating $\mathrm{NOTCH} 1^{12-15}$. To ascertain whether the above-observed effects depend on the regulation of LINC00152 on the miR-139-5p/NOTCH1 pathway, we first evaluated the relationship among LINC00152, miR139-5p and NOTCH1 using luciferase assays. As a result, the overexpression of LINC00152, but not the vector control, blocked the inhibitory effect of miR-139-5p on the relative luciferase expression of pLuc-NOTCH1-3' UTR (Fig. 5e). These results confirmed that LINC00152 abolishes the miR-139-5p-mediated repressive activity on NOTCH1 by competitively binding miR-139-5p. In addition, LINC00152 knockdown significantly reduced the endogenous NOTCH1 expression in CRC cells (Fig. 5f). In contrast, NOTCH1 expression was obviously increased in LINCO0152 overexpressing CRC cells (Fig. 5f). A positive relationship was also observed between the levels of NOTCH1 and LINCOO152 in CRC tissues (Fig. 5g). These data demonstrate that LINC00152 can regulate NOTCH1 activity by sponging miR-139-5p both in CRC cell lines and clinical CRC tumors.

\section{LINC00152 exerts tumor-promoting function in CRC by regulating the miR-139-5p/NOTCH1 axis}

Both miR-139-5p and NOTCH1 could regulate cell growth, apoptosis, and invasion in $\mathrm{CRC}^{12-18,22}$. To investigate whether LINCO0152 exerts tumor-promoting functions in CRC by modulating the miR-139-5p/
NOTCH1 axis, we first checked the effects of miR-139-5p and NOTCH1 on LINC00152-induced cell proliferation, and observed that miR-139-5p overexpression or NOTCH1 knockdown blocked the LINC00152-induced CRC cell growth (Fig. 6a). We then evaluated the effects of miR-139-5p/NOTCH1 signaling on the LINC00152induced 5-FU resistance in CRC cells. As shown in Fig. 6b, ectopic miR-139-5p expression or NOTCH1 knockdown significantly reversed the LINC00152-induced 5-FU resistance and counteracted the apoptosis-inhibiting effects of LINC00152 in CRC cells (Fig. 6c). In addition, the increased cell mobility in LINC00152 overexpressing CRC cells was also reversed by miR-139-5p overexpression or NOTCH1 knockdown (Fig. 6d). Altogether, these data demonstrate that LINCO0152 exerts tumorpromoting functions in $\mathrm{CRC}$, at least partly, through sponging miR-139-5p and then regulating NOTCH1.

\section{Discussion}

In this study, we observed that LINC00152 expression is obviously increased in clinical CRC tissues, and is correlated with tumor stage and poor patient survival. Functionally, we revealed that LINC00152 promotes CRC growth, metastasis, and induces 5-FU resistance. Moreover, we further demonstrated that LINC00152 executes tumor-promoting functions by sponging miR-139-5p and then modulating NOTCH1 in CRC.

Numerous studies have revealed varied regulatory roles of lncRNAs in human diseases, especially in tumorigenesis and development ${ }^{23}$. For example, our previous work revealed that $U C A 1$ could promote cell proliferation and 5 -FU chemoresistance in CRC via competitively inhibiting miR-204-5 $\mathrm{p}^{24}$. LINC00152 is recently identified cancerrelated lncRNA that play oncogenic roles in several kinds of human cancers, especially in digestive tract tumors ${ }^{4-9}$, 
${ }^{11}$. Yue et al. ${ }^{19}$ reported that LINC00152 expression is increased in CRC. Interestingly, in contradictory to their conclusions, a recently published work demonstrated that LINC00152 is downregulated in CRC, inhibits viability and promotes apoptosis of CRC cells ${ }^{25}$. Here, we demonstrated that LINC00152 expression was obviously increased in CRC and correlated with patient's survival, which was also observed by Yue et al. ${ }^{19}$. Our detailed functional studies revealed the promoting effects of LINC00152 on CRC growth and metastasis, which is coincident with the oncogenic role of LINCO0152 in $\mathrm{GC}^{4-7}$, liver cancer ${ }^{8}$, gallbladder cancer ${ }^{9,26}$, and clear cell renal cell carcinoma ${ }^{27}$. In addition, we also showed that LINC00152 confers resistance to 5-FU-induced apoptosis, which was similar to that reported by Yue et al. ${ }^{19}$. In their study, Yue et al. demonstrated that LINC00152 works as a ceRNA of miR-193a-3p to induce oxaliplatin resistance. These data demonstrate that LINC00152 is a key lncRNA with extensive tumor-promoting functions in human cancers.

Several studies have reported that LINC00152 promotes tumor development and progression by regulating several key tumor-related pathways, including EGFR, mTOR, and PI3K/AKT signaling, ${ }^{4,}$, 9. Recent studies revealed a new

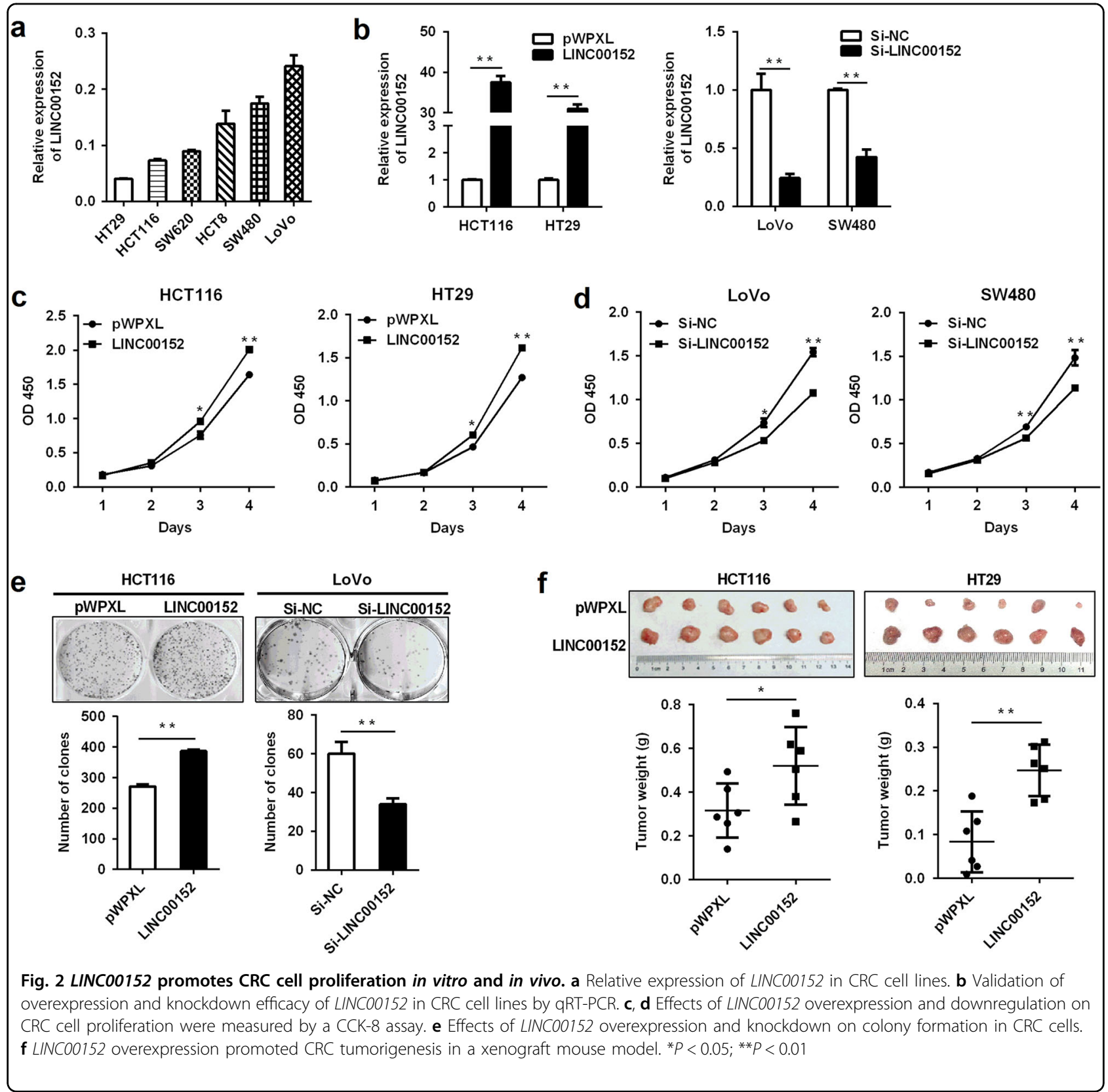




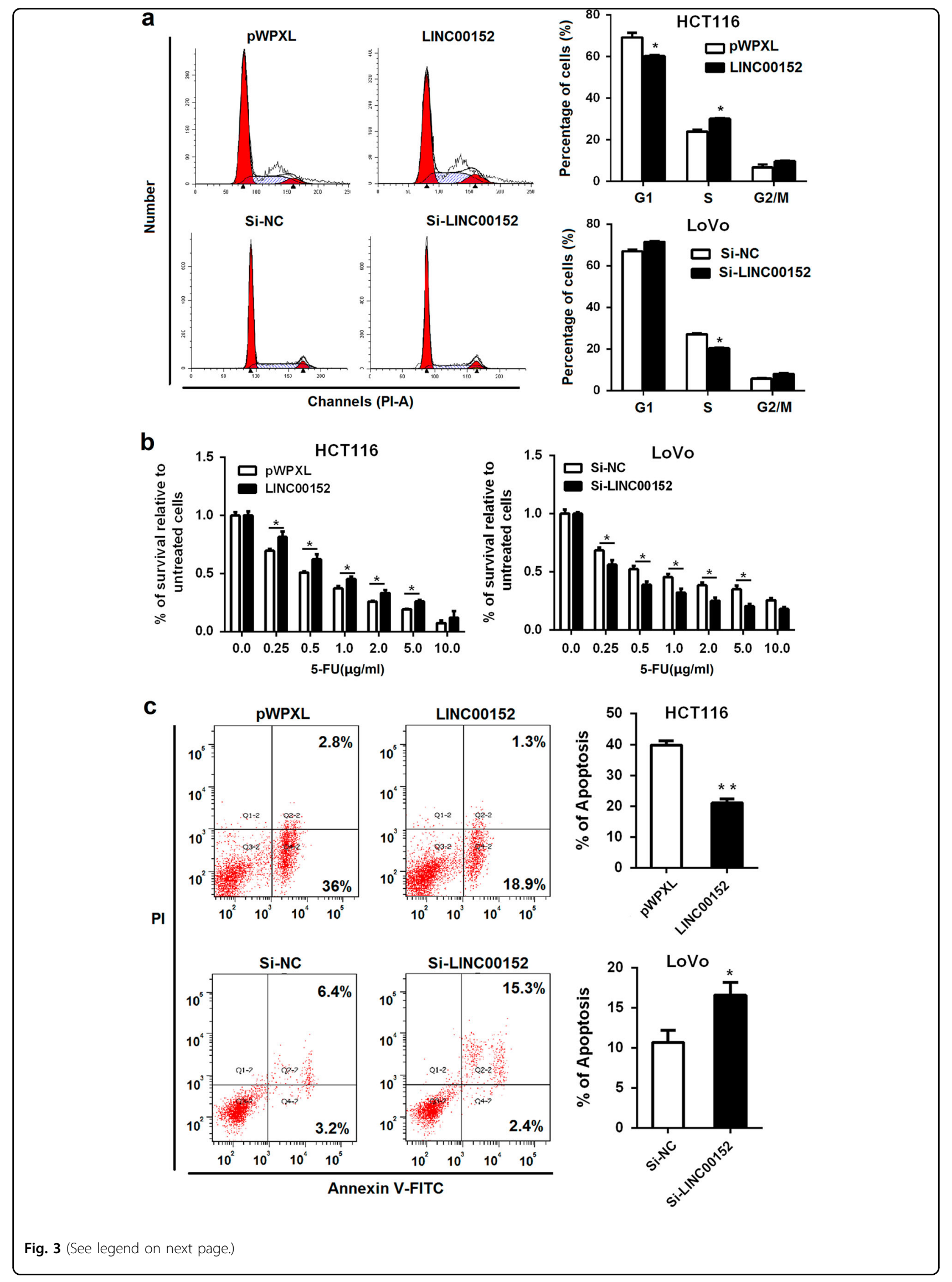


mechanism of lncRNA by acting as ceRNA ${ }^{20}$. In this situation, lncRNAs can block the repression of miRNA on its target genes by competitively binding their common MREs $^{28}$. LINC00152 could bind several miRNAs in cancer cells, including miR-138, miR-376c-3p, and miR-193a$3 \mathrm{p}^{19,25,26}$, suggesting that ceRNA is a key mechanism by which LINC00152 regulates tumorigenesis and development.

Due to the upregulation and tumor-promoting role of LINC00152 in CRC, it is reasonably concluded that LINC00152 promotes CRC development and progression by inhibiting tumor suppressive miRNAs. Based on previous works by us and other researchers, miR-139-5p levels are markedly reduced in CRC, and has exact opposite functions to those of LINC00152 ${ }^{12-18}$. MiR-139$5 p$ can repress CRC growth, metastasis, and chemoresistance by regulating several genes, such as NOTCH1, BCL2, and AMFR ${ }^{12-18}$. MiR-139-5p was reported to play a suppressive role in other cancers, including gastric, breast, and hepatocellular carcinoma ${ }^{29}$. As a key member of the $\mathrm{NOTCH}$ family, NOTCH1 is frequently upregulated in human cancers, including $\mathrm{CRC}^{30}$. Previous researches have proved that miR-139-5p can regulate CRC growth, metastasis, stemness, and chemoresistance via targeting $\mathrm{NOTCH} 1^{12-15,31}$. We speculated that LINCOO152 exerts its functions by regulating the miR-139-5p/NOTCH1 pathway. As expected, both the luciferase and RIP assays confirmed the binding of LINC00152 to miR-139-5p.
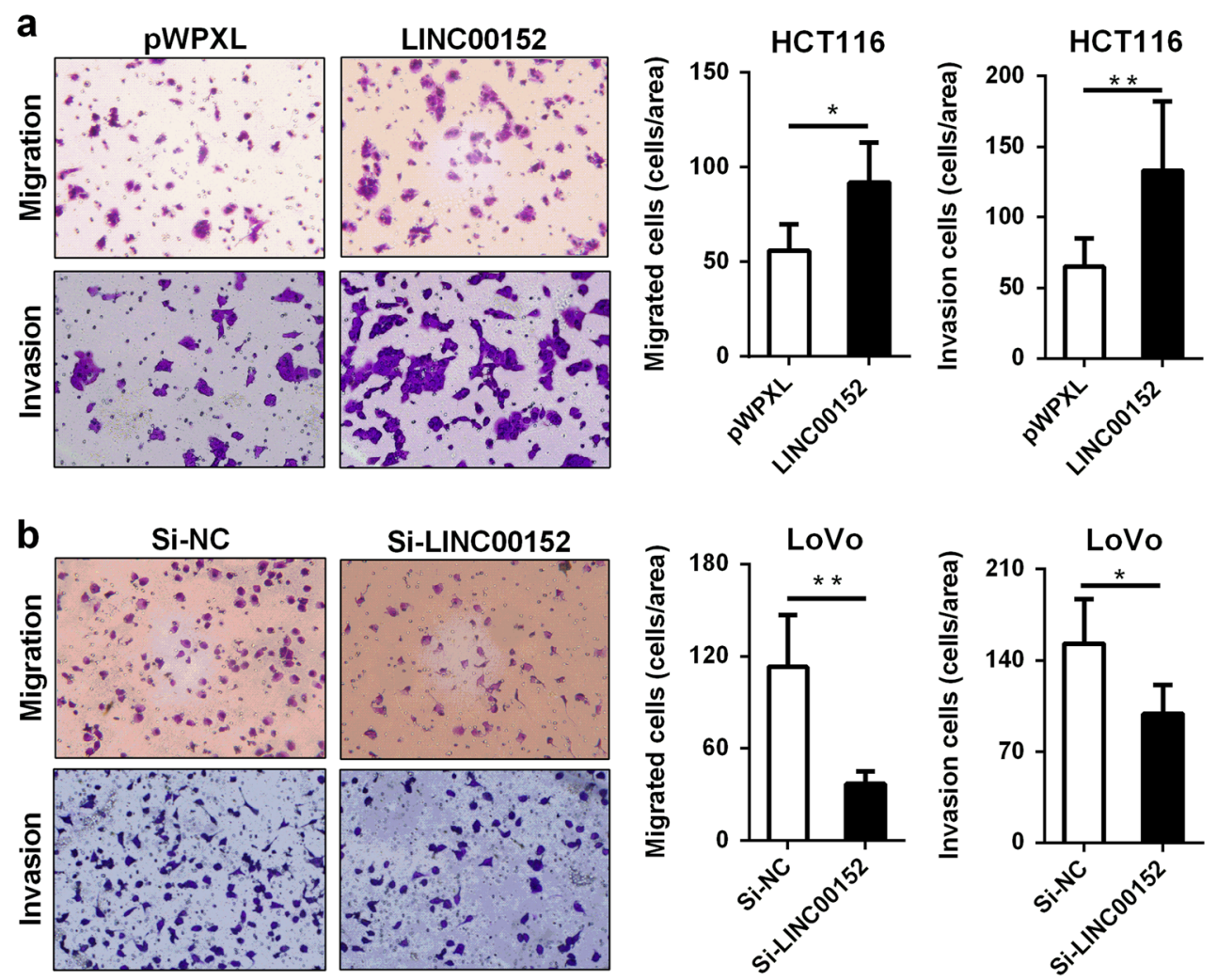

Fig. 4 LINC00152 promotes cell migration and invasion in CRC cells. a, b Representative images and bargraphs depicting the migration and invasion ability of CRC cells with overexpressed or silenced LINC00152. ${ }^{*} P<0.05 ;{ }^{*} P<0.01$

(see figure on previous page)

Fig. 3 LINC00152 promotes cell cycle progression and confers resistance to 5-FU-induced apoptosis. a Cell cycle analyses were performed in HCT116 cells transfected with pWPXL-LINC00152 and pWPXL, or LoVo cells transfected with si-LINC00152 and si-NC. b LINC00152 decreased the sensitivity of CRC cells to 5-FU. The IC50 of LINC00152-overexpressed HCT116 cells was significantly higher than that of the control (0.836 vs. 0.279 $\mu \mathrm{g} / \mathrm{ml})$, and the IC50 of LINC00152-silenced LoVo cells was lower than that the control $(0.576 \mathrm{vs} .0 .960 \mu \mathrm{g} / \mathrm{ml})$. c Cell apoptosis analyses were performed in cell lines with LINC00152 overexpression or knockdown. ${ }^{*} P<0.05 ;{ }^{*} P<0.01$ 


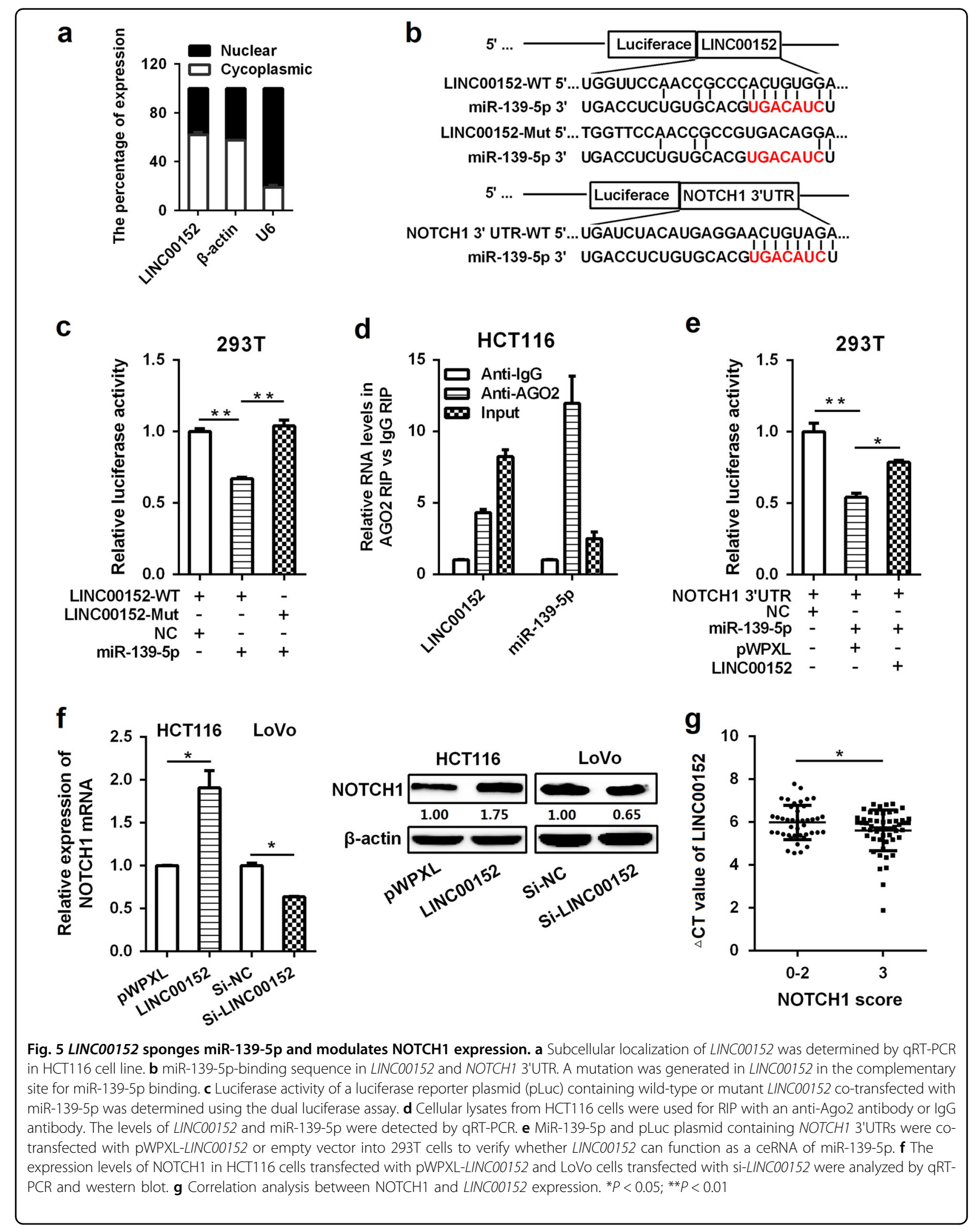




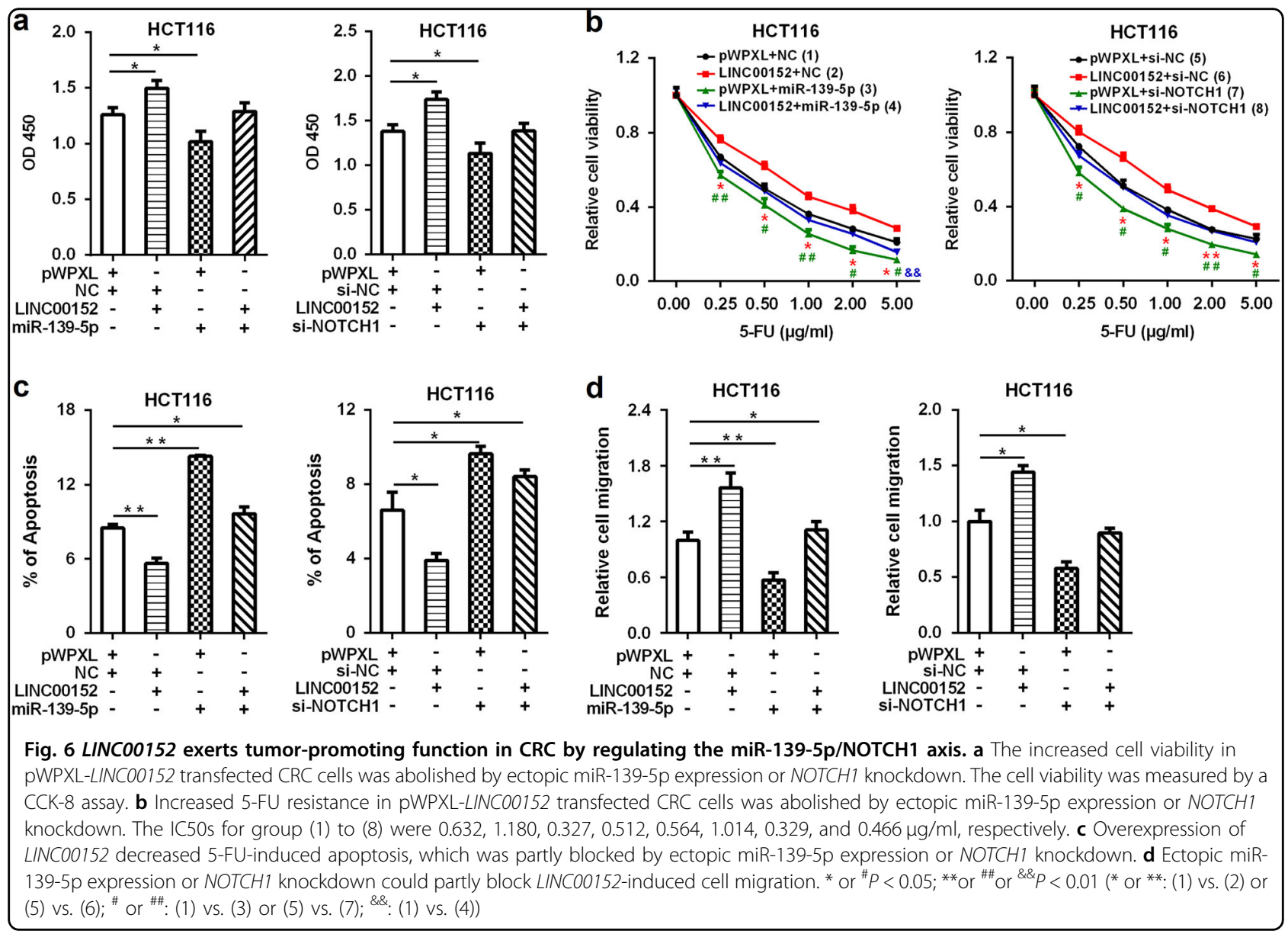

Subsequent functional and mechanistic assays proved that LINC00152 regulates CRC development, progression, and drug resistance by competitively sponging miR-139-5p and then restoring NOTCH1 activity.

In summary, our work shows that LINC00152 is upregulated in CRC, correlated with patients' survival and appears to be a potential biomarker for predicting chemoresistance. LINCO0152 contributes to the tumorigenesis, progression, and chemoresistance of CRC by inhibiting miR-139-5p, uncovering a novel ceRNA network of LINC00152/miR-139-5p/NOTCH1 in CRC cells. These data suggest that targeting LINCO0152 may be a promising therapeutic strategy for CRC.

\section{Materials and methods Clinical samples}

A total of 108 paired human CRC tissues and NCTs were collected with informed consent at Affiliated Hospital of Jiangnan University, and the detailed patient information are shown in Table 1. This study was carried out under the permission of the Clinical Research Ethics Committees of Affiliated Hospital of Jiangnan University.

\section{Cell lines}

HEK-293T and six CRC cell lines (HCT8, HT29, LoVo, HCT116, SW480, and SW620) were obtained from the American Type Culture Collection. These cells were maintained in Dulbecco's modified Eagle's medium supplemented with $10 \%$ fetal bovine serum (Gibco, USA) and have been recently authenticated.

\section{RNA isolation and quantitative reverse transcription (RT)- PCR assays}

Total RNA was isolated with RNAiso Plus (Takara, Japan). Cytoplasmic and nuclear RNA was purified using PARIS $^{\text {TM }}$ Kit (Ambion, USA). Complimentary DNA was synthesized using the HiFiScript 1st Strand cDNA Synthesis Kit (CWBIO, China). Real time RT-PCR was performed using an UltraSYBR Mixture (CWBIO).

\section{Vector construction and siRNA}

LINC00152 was synthesized at GENEray Biotechnology (China) and was inserted into the lentivirus vector pWPXL. The fragment of LINC00152 with miR-139-5pbinding site and the NOTCH1 3'UTR were cloned into 
pLuc. The LINC00152 with the mutated seed sequence of miR-139-5p was constructed by an overlap extension $\mathrm{PCR}^{32}$. The primers used in vector construction are shown in Supplementary Table 1. The siRNAs of LINC00152 and NOTCH1 were purchased from GenePharma (China).

\section{Generation of cell lines with stable overexpression of LINC00152}

HEK-293T cells were transfected with pWPXLLINC00152 (or pWPXL), pMD2G, and ps-PAX2 plasmids using Lipofectamine 2000 (Invitrogen, USA). These virus particles were centrifuged and filtered to infect HCT116 and HT29 cells to generate corresponding stable cells.

\section{Cell proliferation and colony formation assays}

Cell Counting Kit 8 (CCK-8, Beyotime, China) was used to measure cell viability. A colony formation assay was performed as we previously described ${ }^{33}$.

\section{Cell cycle and apoptosis analyses}

The cell cycle and apoptosis analyses of LINC00152overexpressed and silenced CRC cells were applied using the Cell Cycle and Apoptosis Detection Kit purchased from CWBIO.

\section{Cell migration and invasion assay}

Transwell assays were performed to measure cell migration and invasion using Boyden chambers (8-mm pore size, BD Biosciences) as we previously described ${ }^{33}$.

\section{Xenograft tumor assay}

Twenty-four male athymic nude BALB/c mice at 5 weeks of age were randomly divided into four groups, and the number of mice is determined according to prior experience of our laboratory. HCT116 cells stably expressing LINCO0152 or the bank vector were subcutaneously injected into flank of nude mouse. Four (HCT116) or six weeks (HT29) after injection, these mice were sacrificed to measure the growth of subcutaneous tumors. The investigator was blinded to group allocation during the experiments. All animal experiments were approved by the Clinical Research Ethics Committees of our Hospital.

\section{Luciferase reporter assay}

HEK-293T cells were co-transfected with pLuc, pRLCMV, miR-139-5p mimics (negative control, NC), and pWPXL-LINC00152 (pWPXL). These cells were then assayed for luciferase activity using the Dual-Luciferase ${ }^{\circ}$ Reporter Assay System (Beyotime, China).

\section{RNA Immunoprecipitation (RIP) assay}

A RIP assay was performed using the EZ-Magna RIP Kit (Millipore, USA) as we previously described ${ }^{24}$.

\section{Western blotting}

Total protein was separated by $8 \%$ (or $10 \%$ ) sodium dodecyl sulfate polyacrylamide gel electrophoresis and transferred to a PVDF membrane. After blocking with non-fat milk, the polyvinylidene difluoride membrane was incubated with a rabbit anti-human NOTCH1 antibody (1:1000, 20687-1-AP, Proteintech, USA) or a mouse anti$\beta$-actin antibody (1:1000, AA128, Beyotime, China).

\section{Statistical analyses}

Data were presented as the mean \pm s.d. Student's $t$-test, the Mann-Whitney $U$-test and the $\chi 2$ test were performed to analyze the differences among different groups. The differences in survival rates were determined by the Kaplan-Meier method and compared by the log-rank test. HRs and $95 \%$ CIs were calculated by a Cox proportional hazards model. $P$-values $<0.05$ were considered statistically significant.

\section{Acknowledgements}

The study was supported by grants from National Natural Science Foundation of China (81672328, 81602033, 81272299, and 81301784), Natural Science Foundation of Jiangsu Province (BK20150004 and BK20151108), Medical Key Professionals Program of Jiangsu Province, Fundamental Research Funds for the Central Universities (JUSRP51710A and NOJUSRP51619B), Medical Innovation Team Program of Wuxi, and Hospital Management Center of Wuxi (YGZXZ1401 and YGZXM1524).

\section{Author details}

${ }^{1}$ Wuxi Cancer Institute, Affiliated Hospital of Jiangnan University, Wuxi, Jiangsu 214062, China. ²Department of Pathology, Affiliated Hospital of Jiangnan University, Wuxi, Jiangsu 214062, China. ${ }^{3}$ Department of Surgical Oncology, Affiliated Hospital of Jiangnan University, Wuxi, Jiangsu 214062, China. ${ }^{4}$ Department of Medical Oncology, Affiliated Hospital of Jiangnan University, Wuxi, Jiangsu 214062, China

\section{Competing interests}

The authors declare no competing financial interests.

Publisher's note: Springer Nature remains neutral with regard to jurisdictional claims in published maps and institutional affiliations.

\section{Supplementary information}

The online version of this article (https://doi.org/10.1038/s41389-017-0008-4) contains supplementary material.

Received: 22 May 2017 Revised: 18 August 2017 Accepted: 21 September 2017

Published online: 28 November 2017

\section{References}

1. Torre, L. A. et al. Global cancer statistics, 2012. CA Cancer J. Clin. 65, 87-108 (2015).

2. Migliore, L., Migheli, F., Spisni, R. \& Coppede, F. Genetics, cytogenetics, and epigenetics of colorectal cancer. J. Biomed. Biotechnol. 2011, 792362 (2011).

3. Xie, X. et al. Long non-coding RNAs in colorectal cancer. Oncotarget 7, 5226-5239 (2016).

4. Zhou, J. et al. Linc00152 promotes proliferation in gastric cancer through the EGFR-dependent pathway. J. Exp. Clin. Cancer Res. 34, 135 (2015).

5. Chen W. M. et al. Long intergenic non-coding RNA 00152 promotes tumor cell cycle progression by binding to EZH2 and repressing p15 and p21 in gastric cancer. Oncotarget 7, 9773-9787 (2016). 
6. Pang, Q. et al. Increased expression of long intergenic non-coding RNA LINC00152 in gastric cancer and its clinical significance. Tumour Biol. 35, 5441-5447 (2014).

7. Li, Q. et al. Plasma long noncoding RNA protected by exosomes as a potential stable biomarker for gastric cancer. Tumour Biol. 36, 2007-2012 (2015).

8. Ji, J. et al. LINC00152 promotes proliferation in hepatocellular carcinoma by targeting EpCAM via the mTOR signaling pathway. Oncotarget 6, 42813-42824 (2015).

9. Cai, Q. et al. Upregulation of long non-coding RNA LINC00152 by SP1 contributes to gallbladder cancer cell growth and tumor metastasis via PI3KJAKT pathway. Am. J. Transl. Res. 8, 4068-4081 (2016).

10. Chen, Q. N. et al. Long intergenic non-coding RNA 00152 promotes lung adenocarcinoma proliferation via interacting with EZH2 and repressing IL24 expression. Mol. Cancer 16, 17 (2017).

11. Tani, H. \& Torimura, M. Identification of short-lived long non-coding RNAs as surrogate indicators for chemical stress response. Biochem. Biophys. Res. Commun. 439, 547-551 (2013).

12. Song, M. et al. MiR-139-5p inhibits migration and invasion of colorectal cancer by downregulating AMFR and NOTCH1. Protein Cell 5, 851-861 (2014).

13. Zhang, L. et al. microRNA-139-5p exerts tumor suppressor function by targeting NOTCH1 in colorectal cancer. Mol. Cancer 13, 124 (2014).

14. Liu, H. et al. miR-139-5p sensitizes colorectal cancer cells to 5-fluorouracil by targeting NOTCH-1. Pathol. Res. Pract. 212, 643-649 (2016).

15. $\mathrm{Xu}, \mathrm{K}$. et al. MiR-139-5p reverses $\mathrm{CD} 44^{+} / \mathrm{CD} 133^{+}$-associated multidrug resistance by downregulating NOTCH1 in colorectal carcinoma cells. Oncotarget 7, 75118-75129 (2016).

16. Li, Q. et al. miR-139-5p inhibits the epithelial-mesenchymal transition and enhances the chemotherapeutic sensitivity of colorectal cancer cells by downregulating BCL2. Sci. Rep. 6, 27157 (2016).

17. Shen, K. et al. MiR-139 inhibits invasion and metastasis of colorectal cancer by targeting the type I insulin-like growth factor receptor. Biochem. Pharmacol. 84, 320-330 (2012).

18. Zou, F. et al. Targeted deletion of miR-139-5 $p$ activates MAPK, NF-kappaB and STAT3 signaling and promotes intestinal inflammation and colorectal cancer. FEBS J. 283, 1438-1452 (2016).

19. Yue, B., Cai, D., Liu, C., Fang, C. \& Yan, D. Linc00152 functions as a competing endogenous rna to confer oxaliplatin resistance and holds prognostic values in colon cancer. Mol. Ther. 24, 2064-2077 (2016).
20. Yang, C. et al. Competing endogenous RNA networks in human cancer: hypothesis, validation, and perspectives. Oncotarget 7, 13479-13490 (2016).

21. Xia, T. et al. Long noncoding RNA associated-competing endogenous RNAs in gastric cancer. Sci. Rep. 4, 6088 (2014).

22. Fender, A. W., Nutter, J. M., Fitzgerald, T. L., Bertrand, F. E. \& Sigounas, G. Notch1 promotes stemness and epithelial to mesenchymal transition in colorectal cancer. J. Cell Biochem. 116, 2517-2527 (2015).

23. Wang, K. C. \& Chang, H. Y. Molecular mechanisms of long noncoding RNAs. Mol. Cell 43, 904-914 (2011).

24. Bian, Z. et al. LncRNA-UCA1 enhances cell proliferation and 5-fluorouracil resistance in colorectal cancer by inhibiting miR-204-5p. Sci. Rep. 6, 23892 (2016).

25. Zhang, Y. H., Fu, J., Zhang, Z. J., Ge, C. C. \& Yi, Y. LnCRNA-LINC00152 downregulated by miR-376c-3p restricts viability and promotes apoptosis of colorectal cancer cells. Am. J. Transl. Res. 8, 5286-5297 (2016).

26. Cai, Q. et al. Long non-coding RNA LINC00152 promotes gallbladder cance metastasis and epithelial-mesenchymal transition by regulating HIF-1alpha via miR-138. Open Biol. 7, 160247 (2017).

27. $\mathrm{Wu}, \mathrm{Y}$. et al. Long non-coding RNA Linc00152 is a positive prognostic factor for and demonstrates malignant biological behavior in clear cell renal cell carcinoma. Am. J. Cancer Res. 6, 285-299 (2016).

28. Rashid, F., Shah, A. \& Shan, G. Long non-coding rnas in the cytoplasm. Genomics Proteomics Bioinformatics 14, 73-80 (2016).

29. Zhang, H. D., Jiang, L. H., Sun, D. W., Li, J. \& Tang, J. H. MiR-139-5p: promising biomarker for cancer. Tumour Biol. 36, 1355-1365 (2015).

30. Chu, D. et al. High level of Notch1 protein is associated with poor overall survival in colorectal cancer. Ann. Surg. Oncol. 17, 1337-1342 (2010)

31. Vinson, K. E., George, D. C., Fender, A. W., Bertrand, F. E. \& Sigounas, G. The Notch pathway in colorectal cancer. Int. J. Cancer 138, 1835-1842 (2016).

32. Huang, Z. et al. MicroRNA-95 promotes cell proliferation and targets sorting Nexin 1 in human colorectal carcinoma. Cancer Res. 71, 2582-2589 (2011).

33. Yin, $Y$. et al. miR-204-5p inhibits proliferation and invasion and enhances chemotherapeutic sensitivity of colorectal cancer cells by downregulating RAB22A. Clin. Cancer Res. 20, 6187-6199 (2014). 